\title{
Shock Mechanism Analysis and Simulation of High-Power Hydraulic Shock Wave Simulator
}

\author{
Xiaoqiu Xu, ${ }^{1,2}$ Han Tao, ${ }^{1}$ and Junwei Han ${ }^{1}$ \\ ${ }^{1}$ School of Mechatronic Engineering, Harbin Institute of Technology, Harbin 150000, China \\ ${ }^{2}$ Department of Mechanics, Harbin University of Science and Technology, Rongcheng Campus, Rongcheng 264300, China \\ Correspondence should be addressed to Xiaoqiu Xu; 13b908020@hit.edu.cn
}

Received 10 May 2017; Accepted 21 June 2017; Published 2 August 2017

Academic Editor: Abdul Qadir Bhatti

Copyright (c) 2017 Xiaoqiu Xu et al. This is an open access article distributed under the Creative Commons Attribution License, which permits unrestricted use, distribution, and reproduction in any medium, provided the original work is properly cited.

\begin{abstract}
The simulation of regular shock wave (e.g., half-sine) can be achieved by the traditional rubber shock simulator, but the practical high-power shock wave characterized by steep prepeak and gentle postpeak is hard to be realized by the same. To tackle this disadvantage, a novel high-power hydraulic shock wave simulator based on the live firing muzzle shock principle was proposed in the current work. The influence of the typical shock characteristic parameters on the shock force wave was investigated via both theoretical deduction and software simulation. According to the obtained data compared with the results, in fact, it can be concluded that the developed hydraulic shock wave simulator can be applied to simulate the real condition of the shocking system. Further, the similarity evaluation of shock wave simulation was achieved based on the curvature distance, and the results stated that the simulation method was reasonable and the structural optimization based on software simulation is also beneficial to the increase of efficiency. Finally, the combination of theoretical analysis and simulation for the development of artillery recoil tester is a comprehensive approach in the design and structure optimization of the recoil system.
\end{abstract}

\section{Introduction}

In modern shooting range construction process, the consumption was significantly increased due to the real firing practice. Four sorts of capability, that is, reliability, security, testability, and supportability, were the critical aims for the examinations [1]. Increasing development quality, shortening test cycle, and saving full-life research cost had been defined as important objectives of improving operational effectiveness of artillery equipment [2]. Since the 1950s, equivalent reliable artillery simulation test method had been initially explored at home and abroad. For example, an artillery fire shock simulation tester equipped with a hydraulic shock cylinder was developed by US researchers [3, 4]. According to the reports, this simulator was characterized by high accuracy, promoted safety performance, and pertinence, which successfully avoided the shortages as mentioned above.

With the fast development of hardware and software resource, computational simulation is gradually introduced in the design of fire shock simulation tester. Yangwu innovatively reported the build of a numerical simulation model of artillery recoil system simulator with applying the classical internal ballistics and systematic dynamic related theories [5].

A further optimization of the simulation model was proposed by Hang and Zhang which considered the load conditions and structure parameters of DS-II artillery recoil system [6]. Changchun et al. developed a virtual recoil tester in MSC in the ADAMS software for investigating a stereotype of ground artillery tester. The simulation of dynamic recoil process was achieved via applying homogeneous experiment design and influence study of shock parameters using Matlab $[7,8]$. Yuliang et al. carried out finite element simulations and test researches of constitutive models of the rubber wave simulators and conducted optimization design of rubber wave simulators [9-13]. Jiao et al. analyzed shock wave characteristics of a hydraulic damper for shock test machine and achieved the influence of the annular gap, the diameter of the orifice pore, and the shock wave under different velocities [14]. Duym et al. carried out evaluation of shock absorber 
models and presented a nonexhaustive overview of shock absorber models [15]. Shams et al. analyzed hydraulic shock absorber valve behavior using CFD-FEA [16]. Jiuhong et al. presented a design method for fluid viscous dampers, derived mathematical model of viscous dampers, and carried out the shock test [17]. Hou et al. proposed a new model for nonlinear viscous fluid dampers with a simple annular orifice and analyzed its fluid dynamics behavior and shear-thinning effects [18-20]. In conclusion, the method where mass block with high velocity impacted artillery muzzle to simulate artillery firing process was proven as a feasible recoil simulation test method [21, 22], and the effects of structure parameters and shock parameters on dynamics and behavior had drawn attentions for hydraulic shock absorber as well as viscous fluid dampers. In order to verify the effectiveness of the simulation method, the similarity of time consequence was an important evaluation method; whatever the test data or the simulation data, both were seen as time consequence. The similarity measuring algorithm included time bend distance [23], slope distance [24], curvature distance [25], and radian distance [26]. Time bend distance was hard to calculate and slope distance and radian distance reflected the relationship within the adjacent time and were short of the adjacent time relevance. The curvature distance algorithm not only reflected the relationship but also expressed the relevance of the adjacent time. Wang et al. carried out credibility evaluation for guidance system dynamic simulation with curvature distance [27]. Yuliang et al. calculated accuracy evaluation of cannon dynamic recoil simulation based on the numerical and shape similarity and verified that rubber shock wave simulator could realize the cannon dynamic recoil motion [28].

In shock simulation tests, the shock wave simulator played an important role in energy transferring and conversion. In addition, it could also achieve the recoil simulation of various cannons under various loading conditions with adjusting the structure parameters. For a regular wave test, a rubber shock wave simulator was usually used. However, practical shock wave was a complex shock curve; traditional rubber shock wave simulator was not fit for practical shock wave.

Therefore, in the current work, a novel high-power hydraulic shock wave simulator was proposed to simulate the real shock wave. In the paper, the shock process and working principle were described and optimization design analysis of reasonable shock's characteristic parameters was achieved. Finally, the similarity evaluation of shock wave simulation was achieved for evaluating the simulation method based on the curvature. The analysis results indicated that the novel high-power hydraulic shock wave simulator could solve the reappearance problem of practical high-power shock wave, which replaced a traditional rubber wave simulator, and also supplied theoretical references for designs of artillery fire shock simulation test.

\section{Structure and Working Principle}

2.1. Principle of the Live Firing Muzzle Shock Test. Breech resultant force $F_{p t}$ that acted on breech axis direction in live firing was an active force that drives artillery recoil motion, which reaches $10^{7} \mathrm{~N}$, and the whole process just requires 5-11 ms; this process belongs to instant strong shock.

$$
m_{h}=\frac{d^{2} x}{d t^{2}}=F_{p t}-F_{R}
$$

where $m_{h}$ was the mass of recoil parts; $x$ was recoil displacement; $t$ was recoil time; $F_{p t}$ was breech resultant force; $F_{R}$ was recoil resistance. Breech resultant force $F_{p t}$ was expressed as formula (2), when the projectile moves in the bore [29].

$$
F_{p t}=\frac{1}{\varphi}\left(1+\frac{1}{2} \frac{m_{\varrho}}{m_{q}}\right) s p,
$$

where $\varphi$ was secondary work calculation coefficient, $m_{\emptyset}$ was explosive payload, $m_{q}$ was projectile mass, $s$ was crosssectional area of line chamber, and $p$ was powder gas pressure, which changes with time and projectile trip. Shock of the projectile on the bore disappeared at the moment when projectile rushed out of the artillery muzzle, which led to a sudden increase of breech resultant force, a phenomenon where transient muzzle-exit $F_{p t g}$ jumped to $F_{g}$ was formed at the beginning of after-effect period.

$$
\begin{aligned}
F_{p t g} & =\frac{1}{\varphi}\left(1+\frac{1}{2} \frac{m_{\varrho}}{m_{q}}\right) s p_{R} \\
F_{g} & =\frac{1}{\varphi}\left(\varphi_{1}+\frac{1}{2} \frac{m_{\varrho}}{m_{q}}\right) s p_{g}
\end{aligned}
$$

where $p_{g}$ was explosive gas's average pressure in artillery bore at the moment of projectile out from the artillery muzzle and $\varphi_{1}$ was secondary coefficient considering projectile rotation and friction. At explosive gas's after-effect period, breech resultant force involves a complex phenomenon of explosive gas flowing out from an artillery muzzle. For convenient calculation, $F_{p t}$ was expressed as formula (4) with exponential empirical formula.

$$
F_{p t}=F_{g} e^{-1 / h}
$$

where $h$ was time constant of breech resultant force damping speed at after-effect period and $t$ was after-effect time at the beginning of after-effect period. In simulation test, an active force that drives artillery recoil motion was $F_{C}$, generated by mass block with high velocity impacting an artillery muzzle.

$$
m_{h}=\frac{d^{2} X}{d t^{2}}=F_{C}-F_{R} .
$$

An alternative model of breech resultant force, where shock force $F_{C}$ replaced breech resultant force $F_{p t}$, played a similar explosive and promoting role for propellant burning. To be certain that shock simulation test possessed higher precision, we need to design right shock parameters and wave simulator structure forms to ensure the equivalence between shock force and breech resultant force. Therefore, for making sure that dynamic behavior of artillery fire shock simulation test was similar to live firing, similar simulation method was chosen, which could generate instant strong shock effect. 


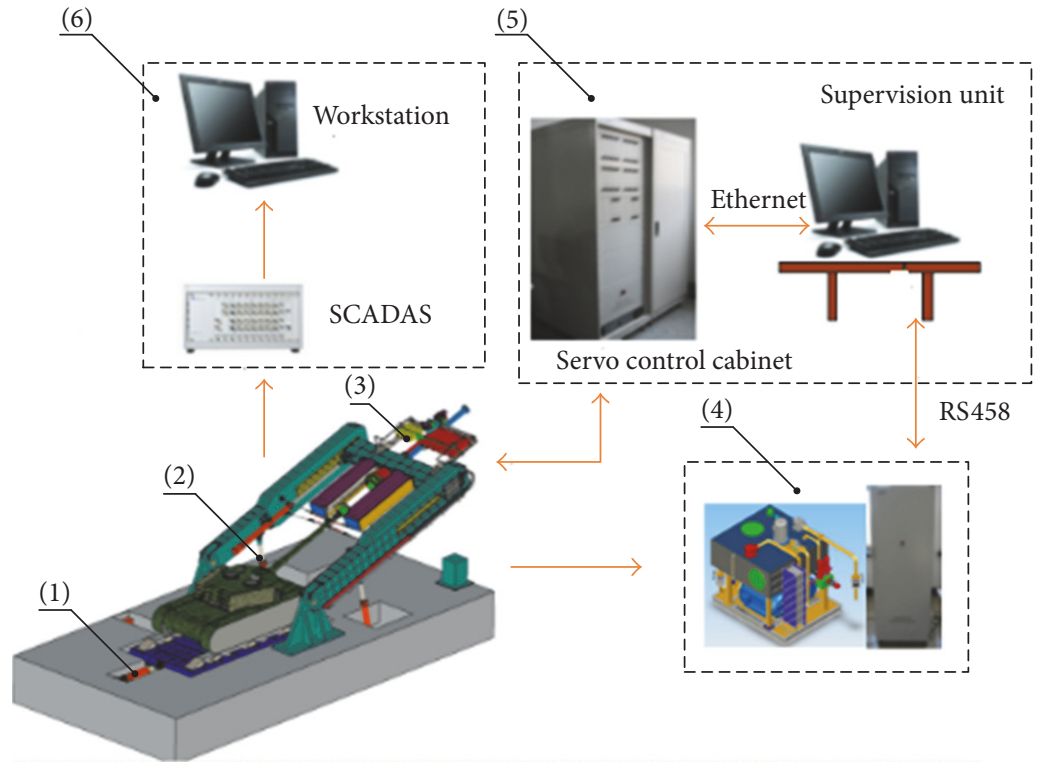

(1) Artillery locating subsystem

(4) Hydraulic power subsystem

(2) Shock locating subsystem

(5) Test control subsystem

(3) Shock simulation subsystem

(6) Parameter measurement subsystem

FIGURE 1: Firing shock simulation test system.

2.2. Shock Simulation Tester Description. Firing shock simulation test system (shown in Figure 1) was a distributing measurement and control system based on industrial Ethernet and fieldbus technology, and the system was composed of six subsystems which were artillery locating subsystem, shock simulation subsystem, test control subsystem, shock locating subsystem, parameter measurement subsystem, and hydraulic power subsystem. In the shock simulation subsystem, shock simulation tester was the key component.

The shock simulation tester used in the firing shock simulation test system was shown in Figure 2 for artillery firing process simulation by computer control. The tester took liquid as storage medium to simulate the shock wave based on controlled hydraulic technology. In the past shock test, the rubber shock simulator was used for simulating the shock wave, and the rubber shock simulator is composed by several rubber blankets with certain stiffness that was calculated for meeting shock wave requirements, and these rubber blankets were installed on subpanel.

Controlling firing rate, firing angle and shock load simulation were realized, which were used to test reliability and durability of the big bore ground and self-propelled and tank artillery. Hydraulic subsystem drove big mass block with shock wave simulator; then the high-velocity big mass block impacted artillery muzzle and simulated the deflagration effect. The simulations made artillery shock test system generate similar recoil motion with artillery live firing.

Shock and restoration processes were shown in Figure 3. Shock wave simulator was accelerated by velocity generator and then impacted muzzle to simulate the required shock wave; after a shock was finished, the shock wave simulator was restated. After the above steps, a shock test was finished, repeating the above process to carry out many experiments.

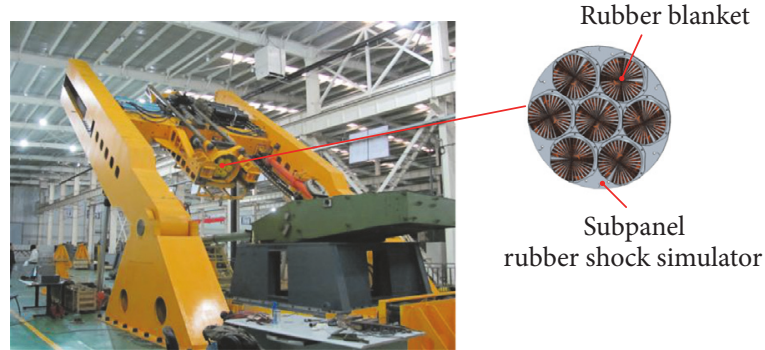

FIGURE 2: Shock simulation tester with rubber shock simulator.

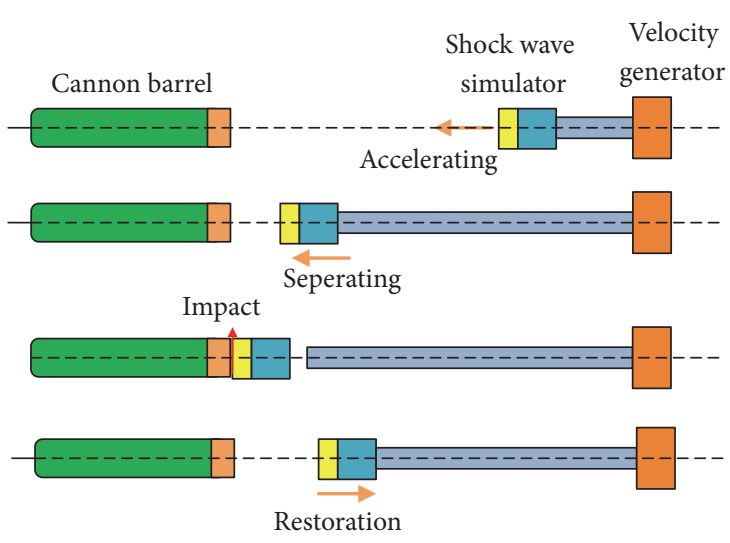

FIGURE 3: Shock and restoration process.

In shock simulation tests, the shock wave simulator was a key component which played an important role in transfer and conversion of shock energy. 


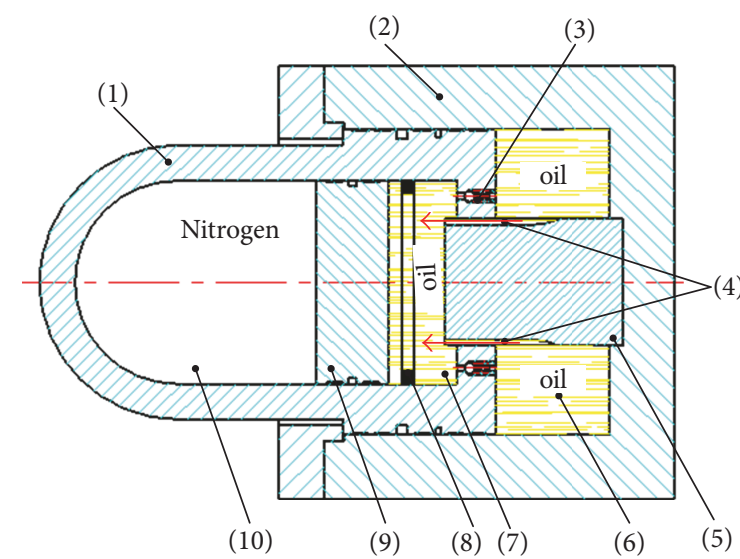
(1) Moving block
(2) Fixed block
(3) One-way check valve
(4) Throttle groove
(5) Throttle spindle
(6) High pressure cavity
(7) Low pressure cavity
(8) Spacing ring
(9) Separated piston
(10) Nitrogen gas cavity

FIGURE 4: Structure stretch of the high-power hydraulic shock wave simulator.

The rubber shock simulator was usually used for the regular shock wave reappearance such as a half-sine shock wave. Additionally, practical shock force wave simulating the breech force had the characteristic of steep prepeak and gentle postpeak relatively (dotted line in Figure 10); the traditional rubber wave simulator was not fit for the simulation. Therefore, for simulating the practical shock force wave and solving the distortion problem, a new idea about highpower hydraulic shock wave simulator was proposed based on hydraulic buffering technology.

2.3. Structure and Principle of the High-Power Hydraulic Shock Wave Simulator. Shock simulation system adopted the principle of the momentum transfer; that is, a shock wave simulator with a certain velocity transferred momentum into cannon barrel during the shock, and the large shock force and the shock acceleration were formed on cannon barrel, and the shock wave could be adjusted by the damping and rigidity of the shock wave simulator. Structure sketch of the high-power hydraulic shock wave simulator was shown in Figure 4.

The inner diameter of the fixed block was $440 \mathrm{~mm}$, and its initial length was $100 \mathrm{~mm}$. The inner diameter of the moving block was $310 \mathrm{~mm}$, and its initial length was $80 \mathrm{~mm}$; there were two throttle holes in the throttle spindle. The piston chambers were fully filled with silicone oil.

The shock wave simulator was accelerated to certain velocity before shock, and then shocking the cannon barrel through the high-power hydraulic shock wave simulator produced instantaneous shock force. During the shock, the moving block moved to the right relative to the fixed block, which led to the oil pressure rise; silicone oil was squeezed to the left side of moving cylinder block through orifice plunger; then isolating piston was pushed to compress Nitrogen for the energy storage. The damping force varied with oil cavity pressure changes.
When an external shock force was applied to the left of the shock wave simulator, the moving block moved toward the right. The oil in the right fixed block chamber was compressed and flew through the throttle gap into the left chamber (as the red arrow shown in Figure 4). In this process, damping force was raised to balance the external shock force.

\section{Coupling Model Building of a High-Power Shock Wave Simulation Test System}

In the high-power shock simulation test, the whole simulation process could be divided into two stages. The sketch of the whole model is shown in Figure 5. In the figure, the model can be considered as two coupling systems: the left one is the recoil system and the right one is the damping system including a damper.

The shock was the first stage; in this process, the rubber pad located on the pounding head of a high-power hydraulic shock wave simulator collides with the canon muzzle and supplies velocity to the cannon barrel and accelerates the canon barrel including recoil buffer device and the cannon suffered shock force from the shock system and the resistance from the recoil system. In the second process, the moving block part will be braked by the damper part of the highpower hydraulic shock wave simulator.

3.1. Model of the Recoil System. $m_{1}$ represents the canon muzzle and it suffers the shock force $F_{C}$ and recoil resistance $R$ in the axis direction; recoil resistance $R$ includes recuperator force $F_{F J}$, recoil brake force $F_{Z T}$, and constant friction $F_{\varphi}$.

$$
R=F_{F J}+F_{Z T}+F_{\varphi} .
$$

Recuperator force could be expressed as follows in a recoil process:

$$
F_{F J}=50000 \times\left(\frac{2.15}{2.15-x_{1}}\right)^{1.3}
$$

where $x_{1}$ is the displacement of $m_{1}$. Recuperator force is a function related to canon recoil displacement. Recuperator force could be expressed as follows in recoil process.

$$
F_{Z T}=\left(1800 \times \frac{150+a_{x}}{a_{x}{ }^{2}}+130\right)\left(\frac{d x_{1}}{d t}\right)^{2},
$$

where $a_{x}$ is equivalent area of recoil brake ventage and $d x_{1} / d t$ is canon recoil velocity.

$$
a_{x}=\left(23-\frac{\pi}{4} d_{x}^{2}\right),
$$

where $d_{x}$ is throttling bar diameter.

Although the corresponding throttling bar diameters under different recoil displacement are different, throttling bar diameter remains constant.

$$
F_{Z T}=1580 \frac{d x_{1}}{d t} .
$$




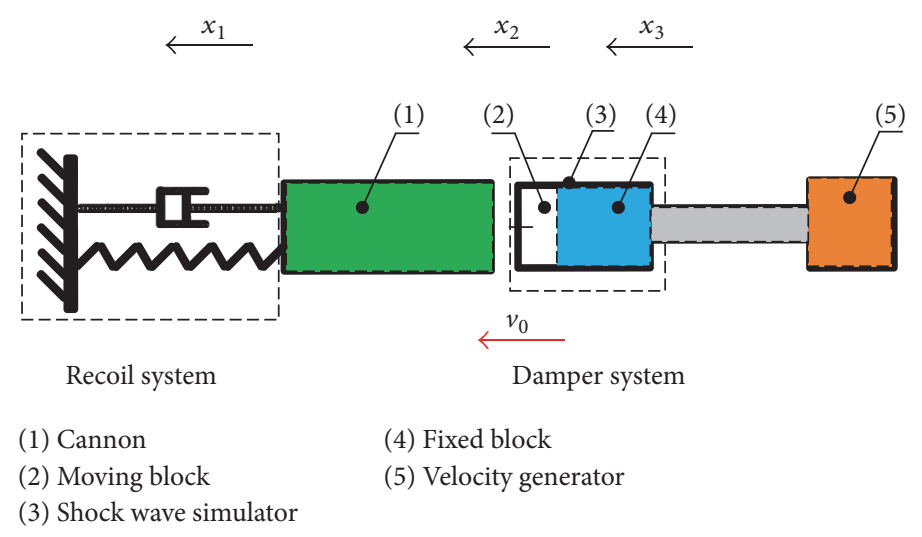

FIGURE 5: Coupling model for the high-power shock simulation test.

Recoil brake force is a function related to canon recoil velocity. Constant friction $F_{\varphi}$ could be expressed as follows:

$$
F_{\varphi}=f_{1} m g \cos \varphi-m g \sin \varphi+F_{0} .
$$

where $f_{1}$ is the friction coefficient between canon recoil and cradle, $\varphi$ is canon firing angle, and $F_{0}$ is the friction generated by plug component of counter-recoil mechanism, which is composed by two parts.

$$
F_{0}=F_{01}+F_{02},
$$

where $F_{01}$ is the friction generated by plug component in recuperator and $F_{02}$ is the friction generated by plug component in the recoil brake. When canon firing angle remains constant, constant friction could be seen as constant value. $F_{\varphi}=10500 \mathrm{~N}$ when canon firing angle $\varphi$ equals $0^{\circ}$.

In conclusion, the force balance equation of the shocked cannon is expressed as follows when firing angle is $0^{\circ}$ :

$$
\begin{aligned}
m_{1} \frac{d^{2} x_{1}}{d t^{2}}= & F_{C}-F_{F J}-F_{Z T}-F_{\varphi} \\
= & F_{C}-50000 \times\left(\frac{2.15}{2.15-x_{1}}\right)^{1.3}-1580 \frac{d x_{1}}{d t} \\
& -10500
\end{aligned}
$$

where $F_{C}$ is the shock force acting on the canon muzzle and $d^{2} x_{1} / d t^{2}$ is recoil acceleration.

3.2. Model of the Damper System. For the shock wave simulator, considering oil compressibility, formula (14) is expressed according to flow continuity function in the damper system.

$$
Q_{j l}+Q_{y s}+Q_{x l}=Q_{z},
$$

where $Q_{j l}$ is the throttled flow, $Q_{y s}$ is the compressed flow, $Q_{x l}$ is the leakage flow, and $Q_{z}$ is the total flow.

$$
Q_{y s}=\frac{A_{1} L-A_{1}\left(x_{2}-x_{3}\right)}{\beta} \frac{d P_{1}}{d t},
$$

where $L$ is the original length, $\beta$ is the bulk elastic modulus of oil, $x_{2}$ is the displacement of the moving block, $x_{3}$ is the displacement of the fixed block, $A_{1}$ is the area of the right end surface of the moving block, and $P_{1}$ is the pressure imposed on the left end surface.

$$
Q_{z}=\left(\frac{d x_{2}}{d t}-\frac{d x_{3}}{d t}\right) A_{1},
$$

where $d x_{2} / d t$ is the velocity of the moving block and $d x_{3} / d t$ is the velocity of the fixed block.

Throttling groove adopts slender hole; flow state is laminar flow because the flow is not free when viscous fluid flows through the throttling groove. The flow of slender hole is related to the viscosity of the fluid. The viscosity of oil changes with its temperature.

When the area $A$ of throttling hole is $\pi d^{2} / 4$, the throttling flow could be expressed as follows:

$$
Q_{j l}=\frac{d^{2}}{(32 \mu l)} A \Delta P=\frac{\pi d^{4} \Delta P}{(128 \mu l)}=\frac{\pi d^{4}\left(P_{1}-P_{2}\right)}{(128 \mu l)},
$$

where $\mu$ is the dynamic viscous, $l$ is the through-flow length, and $d$ is the hole diameter. Formula (18) is achieved by plugging formulas (15), (16), and (17) into formula (14).

$$
\begin{aligned}
& \frac{\pi d^{4}\left(P_{1}-P_{2}\right)}{(128 \mu l)}+\frac{A_{1} Y_{\max }-A_{1}\left(x_{2}-x_{3}\right)}{\beta} \frac{d P_{1}}{d t} \\
& =\left(\frac{d x_{2}}{d t}-\frac{d x_{3}}{d t}\right) A_{1} .
\end{aligned}
$$

The ideal gas adiabatic compression equation is as follows:

$$
\frac{P_{2}}{P_{0}}=\left(\frac{V_{0}}{V_{0}-x_{2} A_{0}}\right)^{k} \text {, }
$$

where $P_{0}$ is the initial pressure in nitrogen gas cavity, $V_{0}$ is the initial volume in nitrogen gas cavity, $A_{0}$ is the inertial effective working area in the nitrogen gas cavity, and $k$ is the adiabatic coefficient. 


\section{Numerical Simulation and Result Analysis}

Using Matlab/Simulink software, firstly, import into the characteristic parameters $\rho, \mu, \beta, k, f_{m c}$ the test parameters $M, m, P_{0}, V_{0}, L$ and the controlled parameters $l, d$ as well as the initial velocity $v_{0}$; then solve the above motion functions according to the fourth-order Runge-Kutta method adopting variable step arithmetic. Shock force curve, acceleration curve, velocity curve, and displacement were received in the high pressure cavity. Finally, model accuracy was verified by the comparison of simulation test and live firing test, and parameters were optimized and corrected. The optimized model could use debugged shock wave simulator for simulating various shock force waves. The specific method was that practical shock force curve was imported as a known condition, throttling area curve was deduced, and required shock force curve was simulated by adjusting the throttling area.

From the building process of mechanical model, the factors affecting shock wave characteristics were mainly divided into three classes: the first class was test parameters including $\rho, \mu, \beta, k$, and $f_{m c}$; the second class was characteristic parameters including $M, m, P_{0}, V_{0}$, and $L$, and the third class was controlled parameters including $d$ and $v_{0}$.

The influences of typical parameters on the shock force wave were analyzed, and these typical parameters included initial velocity $v_{0}$, dynamic viscosity $\mu$, the bulk elastic modulus $\beta$ of oil, and the diameter $d$ of the throttling hole.

4.1. The Influence of Initial Velocity $v_{0}$ on the Shock Behavior. The influence of initial velocity $v_{0}$ on the shock behavior in controlled parameters could be inferred in that the increase of initial velocity would increase the total flow $Q_{z}$ and then affect the shock force according to (17) and (6); the shock force waves were shown in Figure 6 under different initial velocities. In comparison, it was obvious that the shape trend of shock force wave had similar values under different initial velocity $v_{0}$, and the peak value increased with the initial velocity increase, which accorded with the theory analysis as well as the mechanical performances of the shock wave simulator.

4.2. The Influence of Dynamic Viscosity $\mu$ on the Shock Behavior. In characteristic parameters, the dynamic viscosity $\mu$ affected the throttled flow $Q_{j l}$ and the throttled flow $Q_{j l}$ increased with $\mu$ declining according to function (18). Hence, shock force wave was simulated under various dynamic viscosities $\mu$ for describing the influence of the dynamic viscosity $\mu$ on the shock behavior. The curves were shown in Figure 8, and it was obvious that the shape of shock force wave curves changed obviously in the postpeak period under different dynamic viscosity $\mu$ in Figure 7; the shock force decreased seriously with dynamic viscosity $\mu$ declining and the curve's shape fluctuated seriously with dynamic viscosity $\mu$ declining.

4.3. The Influence of the Bulk Elastic Modulus $\beta$ of Oil on the Shock Behavior. According to the building process of mechanical model, the bulk elastic modulus $\beta$ of oil in characteristic parameters affected the compressed flow $Q_{y s}$, and the compressed flow $Q_{y s}$ increased with $\beta$ declining according to function (16). Hence, shock force wave was

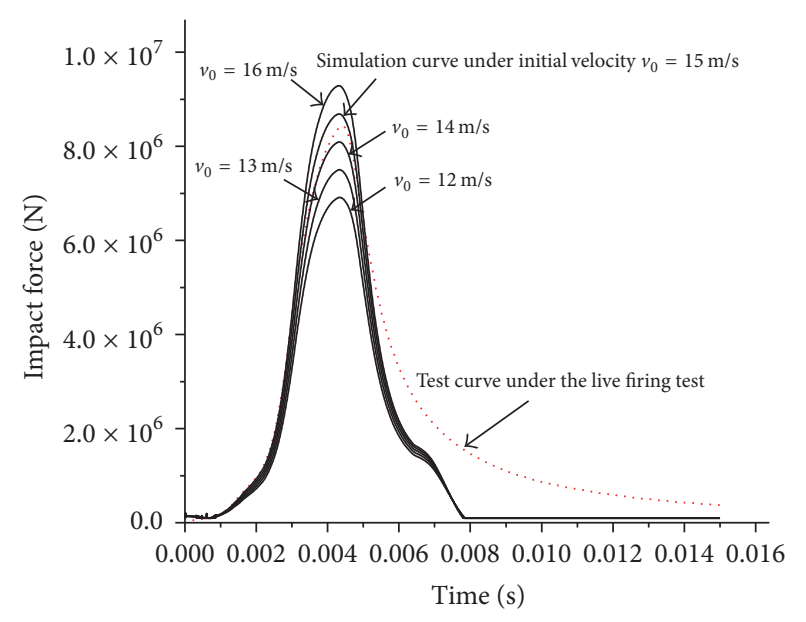

FIGURE 6: Shock force curve of the high-power hydraulic shock wave simulator under various initial velocity $v_{0}$.

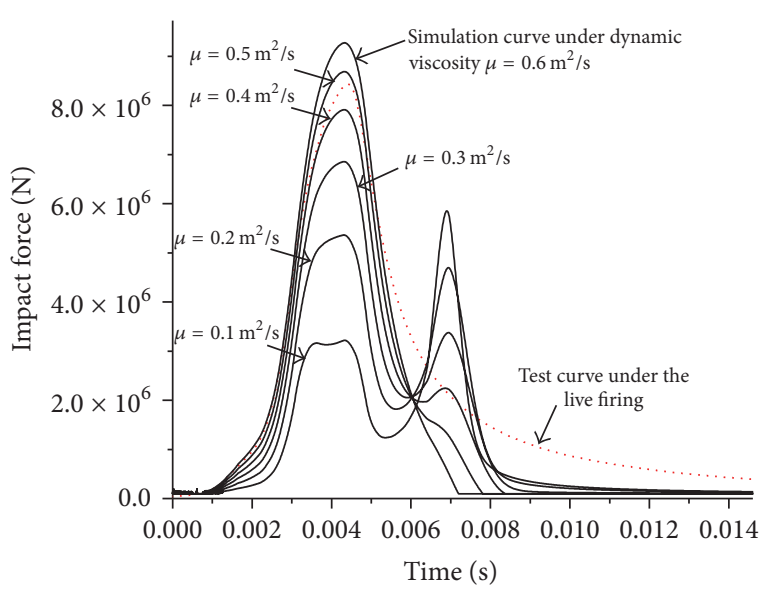

FIGURE 7: Shock force curve of the high-power hydraulic shock wave simulator under various dynamic viscosity $\mu$.

simulated under bulk elastic modulus $\beta$ of oil for describing the influence of the bulk elastic modulus $\beta$ of oil on shock behavior. The comparison curves were shown in Figure 8, and it was obvious that the shape of shock force wave curves changed gently under bulk elastic modulus $\beta$ of oil, the shock force decreased, and the impulse increased with the decline of bulk elastic modulus $\beta$ of oil in Figure 8 .

4.4. The Influence of the Diameter $d$ of the Throttling Hole on the Shock Behavior. In controlled parameters, the diameter $d$ of the throttling hole directly affected the throttled flow $Q_{j l}$ and the throttled flow $Q_{j l}$ increased with the increase of the diameter $d$ of the throttling hole according to function (18). For describing the influence of the diameter $d$ of the throttling hole on the shock behavior, the shock force wave was simulated under varying diameter $d$ of the throttling hole. The curves were shown in Figure 9, and it was obvious that the shape of shock force wave curves changed obviously in the postpeak period, the shock force decreased seriously 


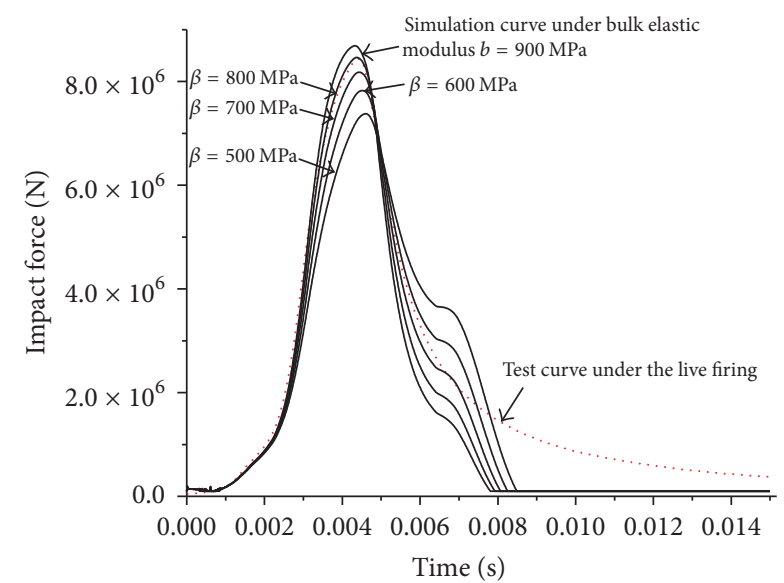

FIGURE 8: Shock force curve of the high-power hydraulic shock wave simulator under various bulk elastic modulus $\beta$ of oil.

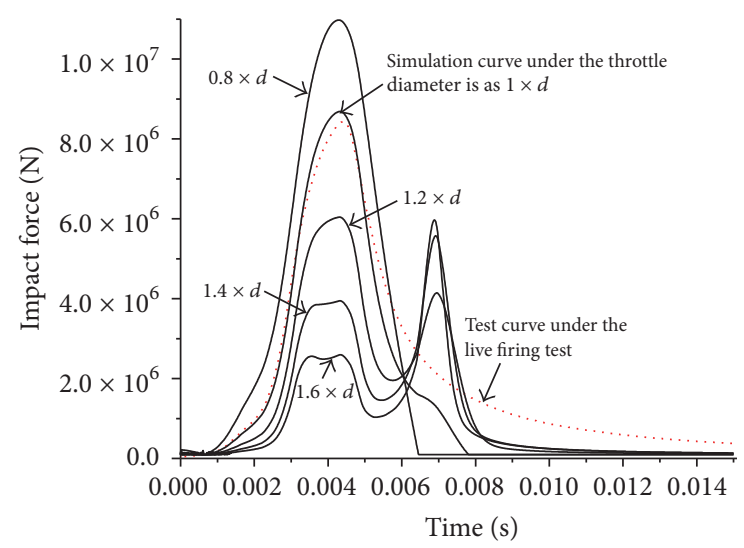

FIGURE 9: Shock force curve of the high-power hydraulic shock wave simulator under varying throttle diameter $d$.

with the increase of the diameter $d$ of the throttling hole, and the wave's shape fluctuated seriously.

By the optimization design analysis of typical shock parameters, a set of reasonable shock parameters were received as shown in Table 1.

The throttle diameter $d$ curve of the optimized model was debugged shock wave simulator for simulating various shock force waves. The specific method was that practical shock force curve was imported as a known condition, throttling diameter $d$ curve was deduced, and the curve required subsection discretization in practical engineering design. Required shock force curve was simulated by the optimization of throttling diameter $d$ curve.

The shock force wave was simulated adopting the optimized shock parameters in Figure 10.

The simulation curve was similar to the test curve under the live firing. It was indicated that the above mathematical model had good precision by optimization and correction of numerical simulation tests under various working conditions, and the high-power hydraulic shock wave simulator adopting the optimized shock parameters realized the shock

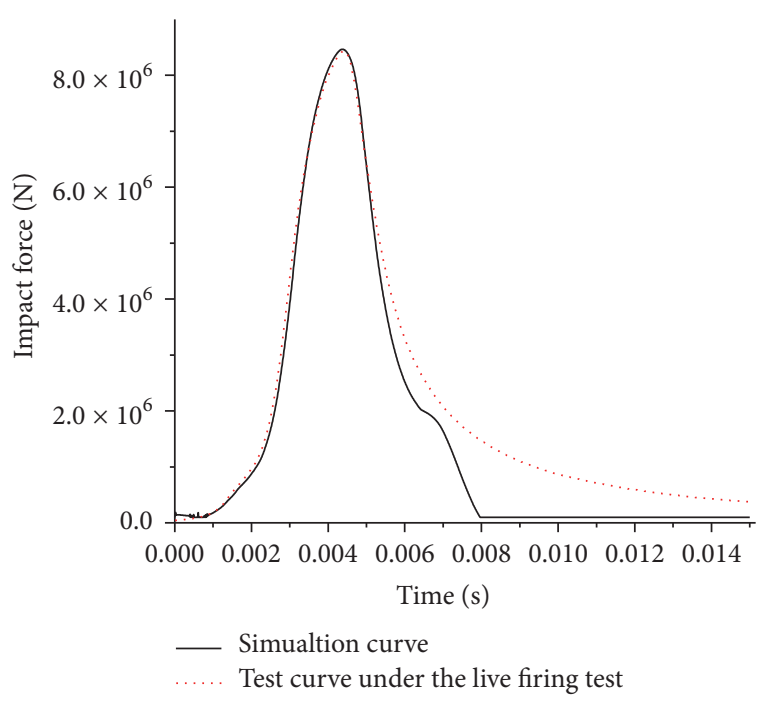

FIGURE 10: Shock force curve comparison generated by the highpower hydraulic shock wave simulator and live firing test, respectively.

wave reappearance of the shock force on artillery muzzle in the live firing test.

\section{Similarity Evaluation}

Using the above data, similarity evaluation was carried out for the simulation method based on the numerical similarity and shape similarity.

5.1. Numerical Similarity. Assume that the test data consequence was $D T=(d t(1), d t(2), \ldots, d t(n))$ and the simulation consequence was $D S=(d s(1), d s(2), \ldots, d s(n))$. Weighted mean of the relative error in the two time sequences was used for expressing numerical similarity. The calculation method was as follows.

When $d t(i)$ and $d s(i)(i=1,2, \ldots, n)$ were both not zero, numerical similarity of each point $s_{1}(i)$ was expressed as follows:

$$
\begin{aligned}
& s_{1}(i) \\
& \quad= \begin{cases}1-\frac{|d t(i)-d s(i)|}{\max (|d t(i)|,|d s(i)|)} & d t(i) d s(i)>0 \\
0 & d t(i) d s(i)<0 .\end{cases}
\end{aligned}
$$

When at least one parameter was zero in $d t(i)$ and $d s(i)$, numerical similarity of each point $s_{1}(i)$ was as follows:

$$
s_{1}(i)= \begin{cases}0 & d t(i)=0 \text { or } d s(i)=0 \\ 1 & d t(i)=0, d s(i)=0 .\end{cases}
$$

Take the ratio of each point's amplitude and all sample points' sum as the weight $\gamma_{i}$ of the point.

$$
\gamma_{i}=\frac{|d t(i)|}{\sum_{i=1}^{n}|d t(i)|} .
$$


TABLE 1: Main shock parameter list.

\begin{tabular}{lccccccc}
\hline Main shock parameter & $M(\mathrm{~kg})$ & $m(\mathrm{~kg})$ & $v_{0}(\mathrm{~m} / \mathrm{s})$ & $\mu\left(\mathrm{m}^{2} / \mathrm{s}\right)$ & $\beta(\mathrm{MPa})$ & $l(\mathrm{~m})$ & $L(\mathrm{~m})$ \\
\hline Value & 3000 & 2540 & 15 & 0.5 & 800 & 0.08 & 0.27 \\
\hline
\end{tabular}

The total numerical similarity $S_{1}$ of the two time sequences was shown as follows:

$$
S_{1}=\sum_{i=1}^{n} s_{1}(i) \gamma_{i}, \quad(i=1,2, \ldots, n)
$$

5.2. Shape Similarity. The shape similarity was calculated according to the curvature distance between the two time sequences [27]; the calculating process was as follows.

Firstly, primitive sequence $X^{(0)}=\left[x^{(0)}(1), x^{(0)}(2), \ldots\right.$, $\left.x^{(0)}(n)\right]$ was translated into nonnegative sequence $X^{(1)}$, and make sure that the sequence shape was constant.

$$
x^{(1)}(i)= \begin{cases}x^{(0)}(i) & \min X^{(0)} \geq 0 \\ x^{(0)}(i)-\min X^{(0)} & \min X^{(0)}<0\end{cases}
$$

For improving the precision and decreasing the calculation amount, the time sequence needs sector-by-sector solution.

$X^{(1)}=\left[X_{1}(1), X_{2}(2), \ldots, X_{m}(1)\right]$ and $X_{j}^{(1)}=\left[x_{j}^{(1)}(1)\right.$, $\left.x_{j}{ }^{(1)}(2), \ldots, x_{j}{ }^{(1)}\left(l_{j}\right)\right]$, where $X_{j}{ }^{(1)}$ was the $j$ th nonnegative subsequence $(j=1,2, \ldots, m), m$ was the number of the subsequences, and $l_{j}$ was the length of $j$ th subsequence.

Nonnegative subsequence was generated according to AGO (Accumulated Generating Operation), and the consequence presents approximate exponential increase law, which decreased the randomness, possessed statistical significance, and kept the relevance of primitive consequence. Nonnegative subsequence $X^{(1)}$ carried out accumulation calculation, generating the consequence $X^{(2)}=\left[X_{1}{ }^{(2)}, X_{2}{ }^{(2)}, \ldots, X_{m}{ }^{(2)}\right]$. Take subsequence $X_{j}^{(2)}$ as example.

$$
x_{j}^{(2)}(p)=\sum_{a=1}^{p} x_{j}^{(1)}(a), \quad(j=1,2, \ldots, m),
$$

where $x_{j}{ }^{(2)}(p)$ was the $p$ th data in $j$ th generated subsequence.

We can get $x_{j}^{(2)}(1)=x_{j}^{(1)}(1)$. Time consequence $X^{(2)}$ carried out subtraction calculation; original nonnegative subsequence $X^{(1)}$ could be achieved as follows:

$$
\begin{aligned}
x_{j}^{(1)}(p)=x_{j}^{(2)}(p)-x_{j}^{(2)}(p-1) & \\
& \left(p=1,2, \ldots, l_{j}\right) .
\end{aligned}
$$

Consequences $X^{(1)}$ and $X^{(2)}$ could be converted into one another, and $X^{(2)}$ reflected change law in consequence $X^{(1)}$, so we can build the model of $X^{(2)}$ to analyze $X^{(1)}$.

For increasing the fitting model accuracy of time consequence, the subsequence $X_{j}^{(2)}$ model was built.

$$
x_{j}^{(2)}(\lambda)=c_{1} e^{\gamma \lambda}+c_{2} \lambda^{3}+c_{3} \lambda^{2}+c_{4} \lambda+c_{5}
$$

where $c_{i}(i=1,2, \ldots, 5)$ and $\gamma$ were model parameters; $\lambda=$ $1,2, \ldots, l_{j}$. The fitting model could represent the exponent, the parabola, and the third power curve according to the value of model parameters $c_{i}(i=1,2, \ldots, 5)$ and $\gamma$, which is the precise expression of the consequence.

Assume that the curvature of each section in time consequence was $K=\left[K_{1}, K_{2}, \ldots, K_{m}\right]$; the curvature was $K_{j}=\left[k_{j}(1), k_{j}(2), \ldots, k_{j}\left(l_{j}\right)\right]$ of each subsequence.

$$
k_{j}(\lambda)=\frac{\left|x_{j}^{(2) \prime \prime}(\lambda)\right|}{\left[1+\left(x_{j}{ }^{(2) \prime}(\lambda)\right)^{2}\right]^{3 / 2}} \quad\left(\lambda=1,2, \ldots, l_{j}\right) .
$$

Assume that the curvatures of the two time consequence were $K=\left[K_{1}, K_{2}, \ldots, K_{m}\right]$ and $\bar{K}=\left[\bar{K}_{1}, \bar{K}_{2}, \ldots, \bar{K}_{m}\right]$; taking subsequences $K_{j}$ and $\bar{K}_{j}$ as example, the shape similarity was calculated: $K_{j}=\left[k_{j}(1), k_{j}(2), \ldots, k_{j}\left(l_{j}\right)\right]$ and $\bar{K}_{j}=\left[\bar{k}_{j}(1)\right.$, $\left.\bar{k}_{j}(2), \ldots, \bar{k}_{j}\left(l_{j}\right)\right]$. follows:

The curvature distance of subsequences $K_{j}$ and $\bar{K}_{j}$ was as

$$
d_{j}=\frac{1}{l_{j}} \sum_{p=1}^{l_{j}}\left|k_{j}(p)-\bar{k}_{j}(p)\right|
$$

Assume that curvature distance threshold value of the two subsequences is th $\mathrm{DC}_{\mathrm{DC}}$. When the curvature distance was less than threshold value, the two subsequences were similar, while when the curvature distance was greater than threshold value $t_{\mathrm{DC}}$, the two subsequences were not similar, where threshold value $\mathrm{th}_{\mathrm{DC}}$ was related to curvature of each point in each subsequence. Use the ratio of all the similar subsequences' length and the consequence total length as measurement of shape similarity of consequence.

$$
S_{2}=\frac{\sum_{j=1}^{n} L(j)}{L(X)}
$$

where $S_{2}$ was the shape similarity of time consequences $X$ and $Y, L(X)$ was the total length of time consequence $X, L(j)$ was the $j$ th similar subsequence of $X$ and $Y$, and $n$ was the number of similar subsequences of $X$ and $Y$.

5.3. Integrated Similarity of Time Consequence. Numerical similarity and shape similarity were, respectively, calculated based on the two time consequences. Based on the multiattribute integrated evaluation theory, we use multiplication relationship to obtain comprehensive similarity.

So the comprehensive similarity of $S$ was as follows between live firing and simulation firing.

$$
S=\sqrt{S_{1} S_{2}}
$$

where $S_{1}$ and $S_{2}$ were, respectively, numerical similarity and shape similarity. 
TABLE 2: Similarity of shock wave simulation.

\begin{tabular}{lccc}
\hline & Subsequence 1 & Subsequence 2 & Total consequence \\
\hline Numerical similarity & 0.95 & 0.78 & 0.894 \\
Shape similarity & 0.92 & 0.73 & 0.857 \\
\hline
\end{tabular}

5.4. Shock Wave Simulation Evaluation. In cannon recoil motion process, recoil time consequence was divided into recoil section and counter-recoil section. Recoil section was from original point to displacement peak point; counterrecoil section was from displacement peak point to the end point. In recoil section, cannon motion velocity was high and had serious vibration and the maximum velocity existed in the section; cannon motion velocity was low and had small vibration. The weights of recoil section and counter-recoil section were, respectively, 0.67 and 0.33 .

The two long subsequences were divided into multiple short subsequences. The length of each short subsequence was 8; numerical similarity and shape similarity of each short subsequence were calculated by the above method; then the numerical similarity and shape similarity of each long subsequence and the total consequence were calculated. Using the data in simulation, the integrated similarity of the shock wave was calculated as in Table 2 .

The evaluation result indicated that the high-power hydraulic shock wave could simulate the dynamic process of live firing accurately. Therefore, the optimization of shock characteristic parameters could improve the simulation accuracy.

\section{Conclusions}

Theoretical analysis and numerical simulation result analysis demonstrated that the high-power hydraulic shock wave simulator could be adopted for simulating the shock force generated on the artillery muzzle; the similarity evaluation of shock wave simulation indicated that high similarity existed between the shock force in simulation test and the practical shock force in live firing test. In other words, the simulation method was reasonable. Finally, the analysis supplied references for the utility of artillery fire simulation technology. The novel high-power hydraulic shock wave simulator replaced a traditional rubber wave simulator to realize practical high-power shock wave reappearance, which also supplied references for the artillery dynamic recoil simulation test.

\section{Conflicts of Interest}

The authors declare that there are no conflicts of interest regarding the publication of this paper.

\section{References}

[1] Z. Fusan, Theory and Practice in Artillery Type Test, National Defence Industry Press, Beijing, China, 2000.

[2] G. Ting, Simulation and Experimental Research on Artillery Firing Shock Simulation Wave form Generator, Harbin Institute of Technology, Heilongjiang Sheng, China, 2014.

[3] M. Cast, "Army test move to 'virtual proving ground," National Defence, vol. 11, pp. 62-64, 2001.
[4] D. Brown, "Simulating firing loads provides flexibility and test repeatability," ADA323489, 1997.

[5] Y. Yangwu, "Numerical simulations of test system and dynamics of the gun recoil," Journal of China Ordnance, vol. 22, no. 2, pp. 152-155, 2001.

[6] X. Hang and Z.-j. Zhang, "Study on dynamic simulation experiments in a artillery dynamic recoil test," Journal of Ballistic, vol. 7, no. 1, pp. 29-33, 1995.

[7] D. Changchun, Y. Yuliang, Q. Yuliang, and C. Kaibo, "Research on the effect of shock parameters in a artillery dynamic recoil test device," Journal of Artillery Launch and Control, vol. 2, pp. 25-28, 2012.

[8] D. Changchun, L. Lin, Z. Jian, and C. Yongcai, "Numerical analysis of artillery dynamic recoil simulation test based on the principle of artillery muzzle shock," Explosion and Shock Waves, vol. 32, no. 3, pp. 323-327, 2012.

[9] Y. Yuliang, Q. Junqi, D. Changchun, and D. Huiyong, "Finite element simulation and test study of the material constitutive model of rubber wave generator," Mechanical Design, vol. 30, no. 9, pp. 86-89, 2013.

[10] Y. Yuliang, Q. Junqi, D. Changchun, S. Yezun, and L. Xiaowei, "Accuracy evaluationof artillery dynamic recoil simulation based on numerical and shape similarity," Journal of Vibration and Shock, vol. 34, no. 10, pp. 157-160, 2015.

[11] Y. Yuliang, Q. Junqi, D. Changchun et al., "Optimization design of artillery dynamic recoil tester," Journal of Vibration and Shock, vol. 33, no. 2, pp. 47-51, 2014.

[12] Y. Yang, C. Di, D. Wu, and C. Liu, "Design on the Theoretical Breechblock Operating Force of Shock Test Bed for artillery Breechblock System," in Proceedings of the 5th International Conference on Information Engineering for Mechanics and Materials (ICIMM '15), Atlantis Press, Huhhot, Inner Mongolia, China, July 2015.

[13] Y. Yang, J. Zheng, C. Di, and J. Chen, "Model and simulation on a shock test bed for gun breechblock system," in Proceedings of the First International Conference on Information Sciences, Machinery, Materials and Energy (ICISMME '15), Atlantis Press, Chongqing, China, April 2015.

[14] S. Jiao, Y. Wang, L. Zhang, and H. Hua, "Shock wave characteristics of a hydraulic damper for shock test machine," Mechanical Systems and Signal Processing, vol. 24, no. 5, pp. 1570-1578, 2010.

[15] S. Duym, R. Stiens, and K. Reybrouck, "Evaluation of shock absorber models," Vehicle System Dynamics, vol. 27, no. 2, pp. 109-127, 1997.

[16] M. Shams, R. Ebrahimi, A. Raouh, and B. J. Jafari, "CFD-FEA analysis of hydraulic shock absorber valve behavior," International Journal of Automotive Technology, vol. 8, no. 5, pp. 615622, 2007.

[17] J. Jiuhong, D. Jianye, W. Yu, and H. Hongxing, "Design method for fluid viscous dampers," Archive of Applied Mechanics, vol. 78, no. 9, pp. 737-746, 2008.

[18] C.-Y. Hou, "Fluid dynamics and behavior of nonlinear viscous fluid dampers," Journal of Structural Engineering, vol. 134, no. 1, pp. 56-63, 2008. 
[19] C.-Y. Hou, "Behavior explanation and a new model for nonlinear viscous fluid dampers with a simple annular orifice," Archive of Applied Mechanics, vol. 82, no. 1, pp. 1-12, 2012.

[20] C.-Y. Hou, D.-S. Hsu, Y.-F. Lee, H.-Y. Chen, and J.-D. Lee, "Shear-thinning effects in annular-orifice viscous fluid dampers," Journal of the Chinese Institute of Engineers, vol. 30, no. 2, pp. 275-287, 2007.

[21] M. Cast, "Army test moves to virtual proving ground," National Defensemble, vol. 11, pp. 62-64, 2001.

[22] L. Lin, D. Changchun, P. Baoqing, and Y. Jiajun, "Test design and optimization of artillery simulation firing test," Journal of Artillery Launch and Control, no. 3, pp. 99-102, 2010.

[23] H. Xiao and Y. Hu, "Data mining based on segmented time warping distance in time series database," Journal of Computer Rearch and Development, vol. 42, no. 1, pp. 72-78, 2005.

[24] L. Jianhai, Z. Jianye, Y. Feng, and P. Quan, "Study of distancemeasuring method in time series based on slope departure," Computer Engineering and Applications, vol. 45, no. 22, pp. 46, 2009.

[25] B.-N. Liu, J.-Y. Zhang, P. Zhang, and Z.-L. Wang, "Similarity search method in time series based on curvature distance," Journal of Electronics and Information Technology, vol. 34, no. 9, pp. 2200-2207, 2012.

[26] Y.-W. Ding, X.-H. Yang, G.-C. Chen, and A. J. Kavs, "Radiandistance based time series similarity measurement," Journal of Electronics and Information Technology, vol. 33, no. 1, pp. 122128, 2011.

[27] F. Wang, X.-G. Liang, Y.-K. Wang, and Q. Zheng, “Credibility evaluation for guidance system dynamic simulation with curvature distance," Journal of System Simulation, vol. 25, no. 8, pp. 1896-1900, 2013.

[28] Y. Yuliang, Q. Junqi, D. Changchun, D. Huiyong, and Z. Xiaoga, "Modeling and firing simulation accuracy assessment of a gunpower-recoil test table," Journal of Vibration and Shock, vol. 33, no. 10, pp. 127-130, 2014.

[29] Z. Xiangyan, Z. Jianguo, and Y. Junrong, Cannon Design Principle, Beijing Institute of Technology Press, Beijing, China, 2005. 


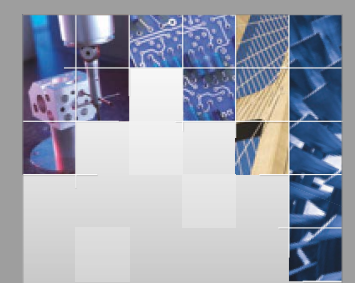

\section{Enfincering}
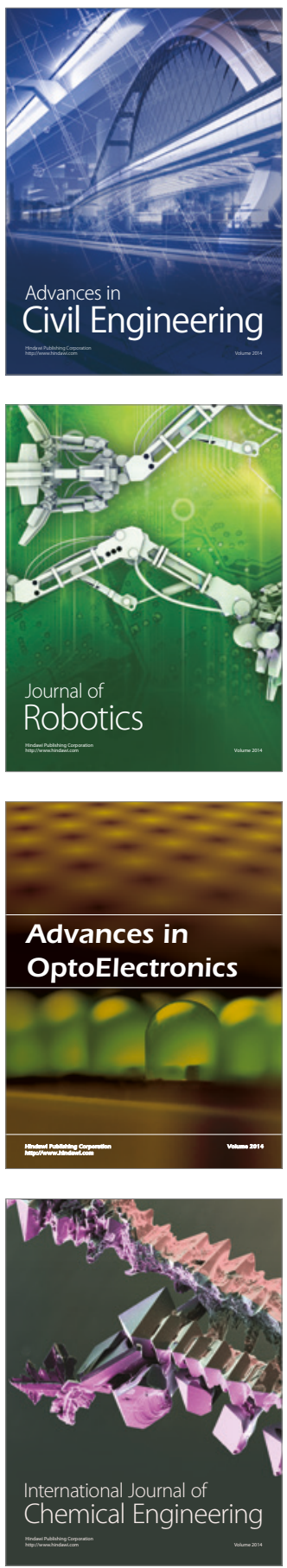

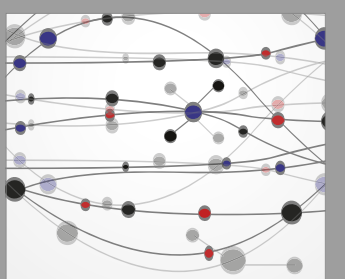

The Scientific World Journal

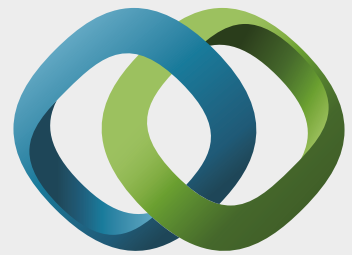

\section{Hindawi}

Submit your manuscripts at

https://www.hindawi.com
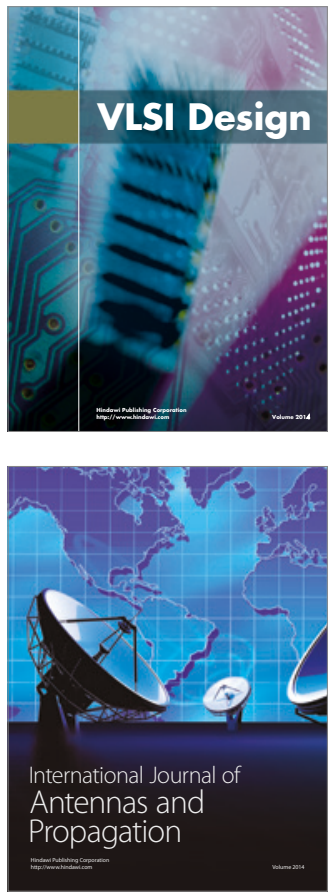

\section{Rotating}

Machinery
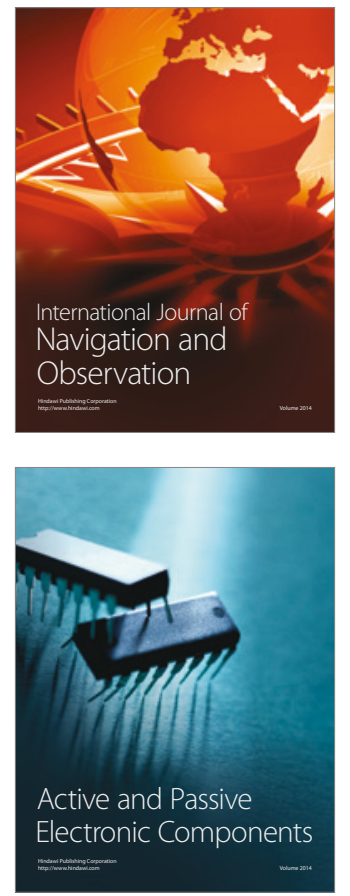
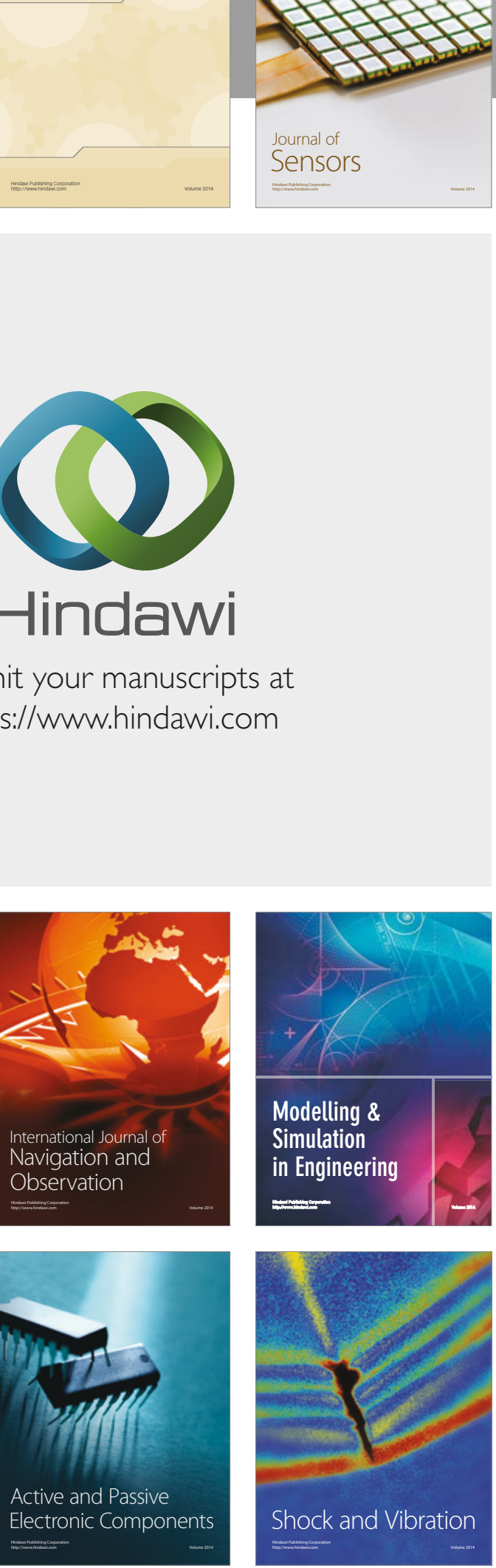
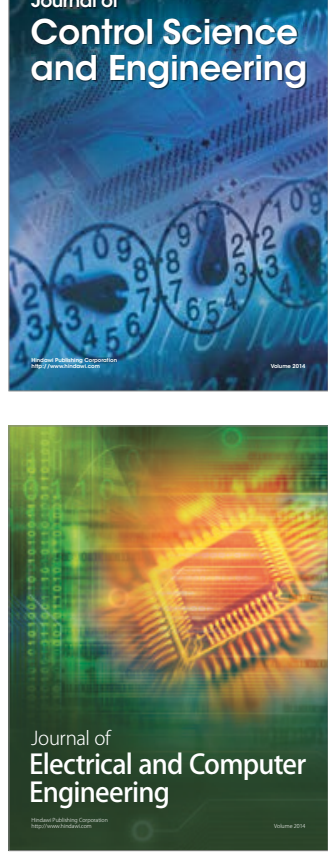

Distributed

Journal of

Control Science

and Engineering
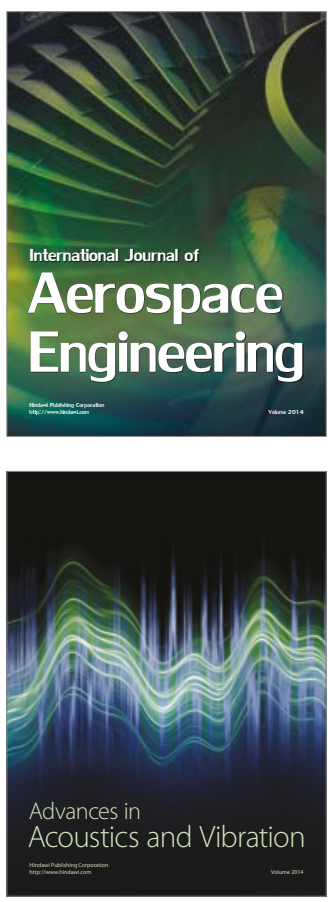

Sensor Networks 